Classification

Physics Abstracts

$71.55 \mathrm{~J}$

\title{
One and two-dimensional quantum localization in GaAs wires of rectangular cross-sections
}

\author{
M. Laviron \\ L.C.R. Thomson-CSF, 91401 Orsay Cedex, France \\ P. Averbuch, H. Godfrin and R. E. Rapp (*) \\ Centre de Recherches sur les Très Basses Températures, CNRS, BP 166 X, \\ 38042 Grenoble Cedex, France
}

(Reçu le 30 juin 1983, révisé le 12 octobre, accepté le 20 octobre 1983)

Résumé. - Nous avons mesuré la variation thermique de la résistance de trois fils gravés d'AsGa (épaisseur : $0,32 \mu \mathrm{m}$; largeurs : 0,$8 ; 2,4$ et $5,4 \mu \mathrm{m}$, longueurs : 400, 400, $200 \mu \mathrm{m}$ ).

On observe la transition entre un régime unidimensionnel à basses températures et un régime à deux dimensions au-dessus de $1 \mathrm{~K}$.

Dans les deux limites, les résultats sont analogues à ceux observés séparément dans des échantillons $1 \mathrm{~d}$ et $2 \mathrm{~d}$ sur d'autres systèmes.

\begin{abstract}
We have measured the thermal behaviour of the resistance of three GaAs wires (thickness : $0.32 \mu \mathrm{m}$, widths : $0.8 ; 2.4$ and $5.4 \mu \mathrm{m}$; lengths : 400,400 and $200 \mu \mathrm{m}$ ) produced by lithographic techniques.

We observe a cross-over between a one-dimensional regime, at low temperatures, and a two-dimensional regime, above $1 \mathrm{~K}$.

In both limits, the results are similar to those observed separately on $1 \mathrm{~d}$ and $2 \mathrm{~d}$ samples in other systems.
\end{abstract}

Both theories of quantum localization, either as a one electron phenomenon $[1,2]$ or as an effect of the disorder through correlations [3, 4], have been obtained for systems whose dimensionality is given a priori. Furthermore the experimental studies have been done mainly on wires [5, 6], films [7] or inversion layers [8], with the exception of one pioneering experiment [9] and of a systematic study in the strong localization regime [10] described by the Mott formulae [11].

In order to clarify the size conditions for which a sample, which a $3 \mathrm{~d}$ object, can be described by a $2 \mathrm{~d}$ or $1 \mathrm{~d}$ model, we have started by measurements of the resistance of wires produced lithographically with the same thickness but different widths. The purpose was to observe the freezing of the lateral degrees of freedom, one after the other when cooling the sample, and the effect of the size of the rectangular cross-section on the cross-over temperature.

$\left({ }^{*}\right)$ Permanent address : Universidade Federal do Rio de Janeiro, Cidade Universitaria, 21910 Rio de Janeiro, Brasil. 
The samples were made with a $0.32 \mu \mathrm{m}$ thick layer grown by vapour phase epitaxy on a semiinsulating gallium arsenide substrate with an undoped buffer layer. This active layer was doped with $10^{17} \mathrm{~cm}^{-3} \mathrm{Si}$ impurities; at low temperatures, this degenerate semiconductor has a Fermi energy $E_{\mathrm{F}} \sim 80 \mathrm{~K}[12,13]$. Ohmic contact pads were made by deposition of germanium nickel and annealing. Wires were defined with an electron beam pattern generator, unwanted GaAs was chemically etched and the etching depth checked by electrical measurements.

The wires were labelled as $a, b$ and $c$ : their widths were : a) $0.8 \mu \mathrm{m}, b) 2.4 \mu \mathrm{m}$; and c) $5.4 \mu \mathrm{m}$ as measured by scanning electron microscopy. A photograph of the thinner wire is given in figure 1 : one can see that, due to the chemical etching, the section of the wire is not quite rectangular. The different wires were all patterned on the same chip and gold thermocompression contacts were made to a TO8 package. The length of the wires was : a) $400 \mu \mathrm{m} ; b) 400 \mu \mathrm{m}$; c) $200 \mu \mathrm{m}$.

The wires were measured as function of temperature from $3.5 \mathrm{mK}$ to $4 \mathrm{~K}$, the chip being in the mixing chamber of a dilution refrigerator. The temperature was determined through a mutual inductance bridge by the magnetic susceptibility of C.M.N. The resistance of the samples was measured with a four wires bridge working at $32 \mathrm{~Hz}$. The sample was protected from ambient radio frequency fields by filters and this was found absolutely necessary for the measurements to have any meaning. Furthermore, as given in figure 3, the apparent resistance was a function of the current in the wire, as discussed below. A more detailed description of the low temperature measurements will be published elsewhere.

Figure 2 is a semilog plot of the conductance (normalized to the one at $4.2 \mathrm{~K}$ ) for three different wires, of three different widths. At $4.2 \mathrm{~K}$, the resistance of the wires was : a) $559.72 \mathrm{k} \Omega$; b) $201.31 \mathrm{k} \Omega$; c) $45.132 \mathrm{k} \Omega$. The minimum current was $10 \mathrm{pA}$ and for temperatures below $100 \mathrm{mK}$ one cannot really speak of conductance, the resulting value being a function of the measuring current. Figure 3 shows, for the thinner wire, the effect of the current.

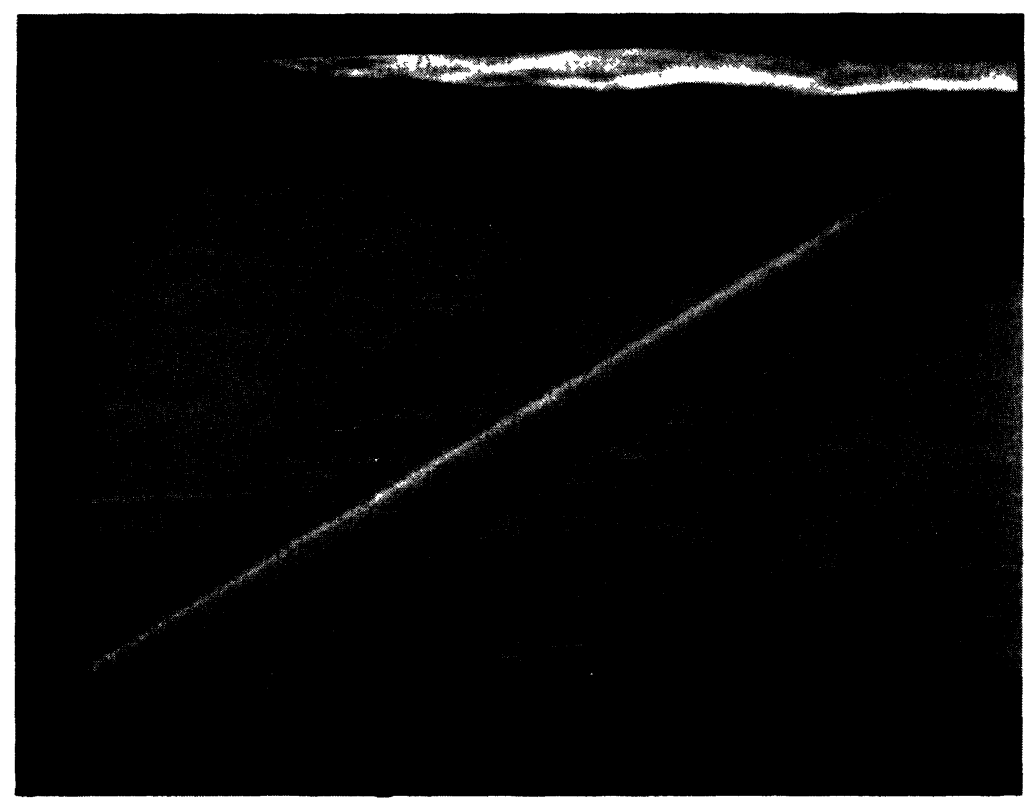

Fig. 1. - Scanning electron microscope photograph of the thinnest wire. One can see the inclination of the edge about the basal plane. 


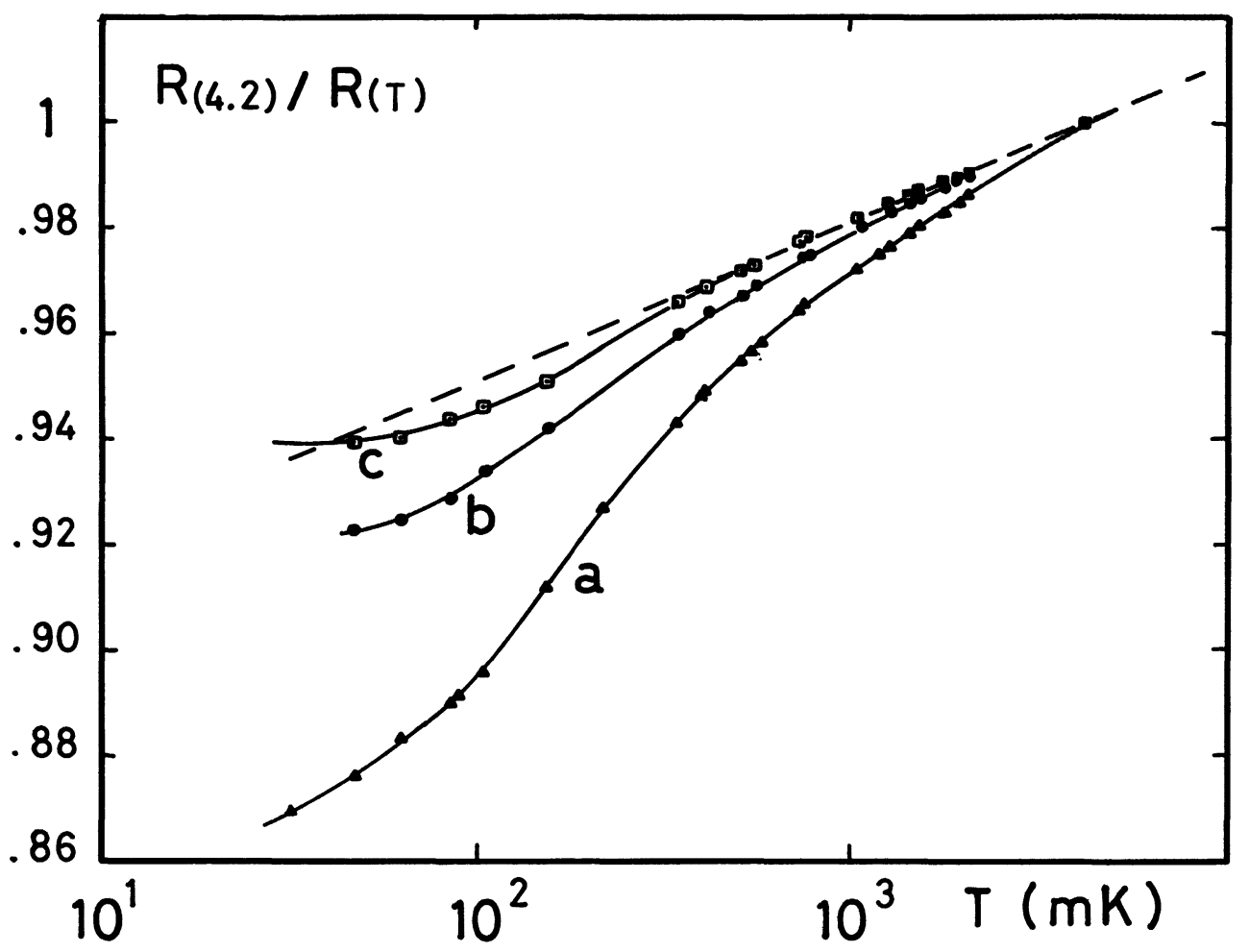

Fig. 2. - Normalized conductance variation for the wires $a, b$ and $c$, showing the $2 \mathrm{~d}$ logarithmic behaviour at high temperatures (dashed line), the cross-over to the 1d regime (see also figure 4), and electron heating effects below $100 \mathrm{mK}$ (see figure 3). Note the shift towards the high temperatures when decreasing the sample thickness from $5.4 \mu \mathrm{m}(c)$, to $2.4 \mu \mathrm{m}(b)$ and $0.8 \mu \mathrm{m}(a) . R(4.2)$ is the resistance at $4.2 \mathrm{~K}$, and $R(T)$ the resistance at the temperature $T$ of the phonons.

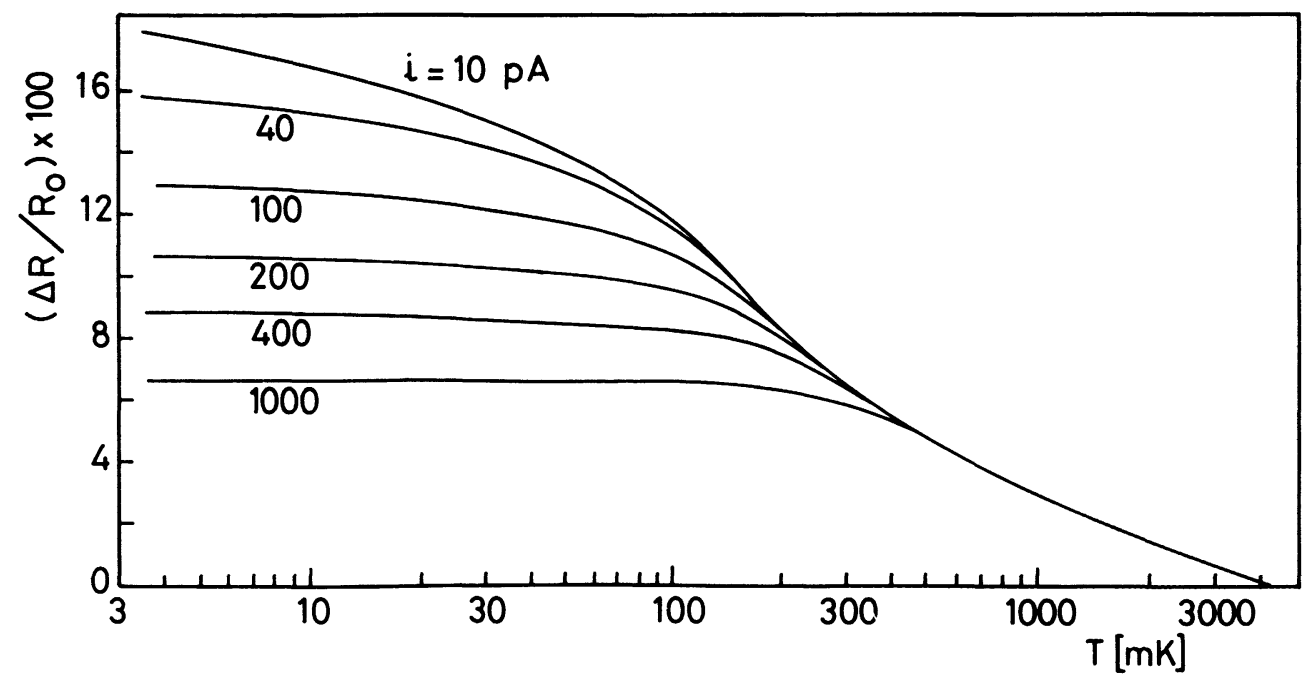

Fig. 3. - Effect of the measuring current on the apparent resistance of wire a. With the present experimental set up, it appears to be unrealistic to get the true resistance below $100 \mathrm{mK} . \Delta R=R(T)-R_{0}$, where $R_{0}$ is the resistance at $4.2 \mathrm{~K}$. 
On the curves of figure 2 one can see, for temperatures higher than $1 \mathrm{~K}$, the logarithmic relation between conductance and temperature; at lower temperatures as long as saturation effects do not appear, the behaviour is consistent with a $T^{-1 / 2}$ law (Fig. 4). These results are similar to those found both in wires and in inversion layers [5-8]. Clearly the two-dimensional effects exist already at $4.2 \mathrm{~K}$, but that is not sufficient to conclude that motion is quenched for a thickness of $0.32 \mu \mathrm{m}$. Due to space charge effect the active part is certainly much thinner.

A comparison of the logarithmic $2 \mathrm{~d}$ behaviour of the $2 \mathrm{~d}$ conductivity $\sigma$ versus temperature with the formula for the one electron model

$$
\sigma=\sigma\left(T_{0}\right)+(\alpha p / 2)\left(e^{2} / \pi^{2} \hbar\right) \ln \left(T / T_{0}\right),
$$

where $\alpha=1$ in the absence of spin-orbit effects, gives $p \sim 0.9$. This value is to be compared with values of 2, 3 or 4 expected from the one electron Anderson localization model [2] with electronphonon interactions, and a value close to 1 when electron-electron interactions are dominant [14].

According to the correlation description $[3,4]$

$$
\sigma=\sigma\left(T_{0}\right)+(1-F)(1 / 2)\left(e^{2} / \pi^{2} \hbar\right) \ln \left(T / T_{0}\right)
$$

then $F$ would have a reasonable value of the order of 0.1 .

The low temperature (1d) behaviour expected from the correlation model [3] is :

$$
\delta C=-(\pi / L)\left(e^{2} / \pi^{2} \hbar\right)\left(2-F^{\prime}\right)\left(\hbar D / 2 k_{\mathrm{B}} T\right)^{1 / 2}
$$

where $C$ is the conductance and $L$ the length of the sample.

The results shown in figure 4 for the thinner wire (a) agree with the $T^{-1 / 2}$ temperature dependence, as well as with the order or magnitude predicted (within a factor of 3 ), the diffusion coefficient being estimated, as usual, by the Einstein relation for the conductivity.

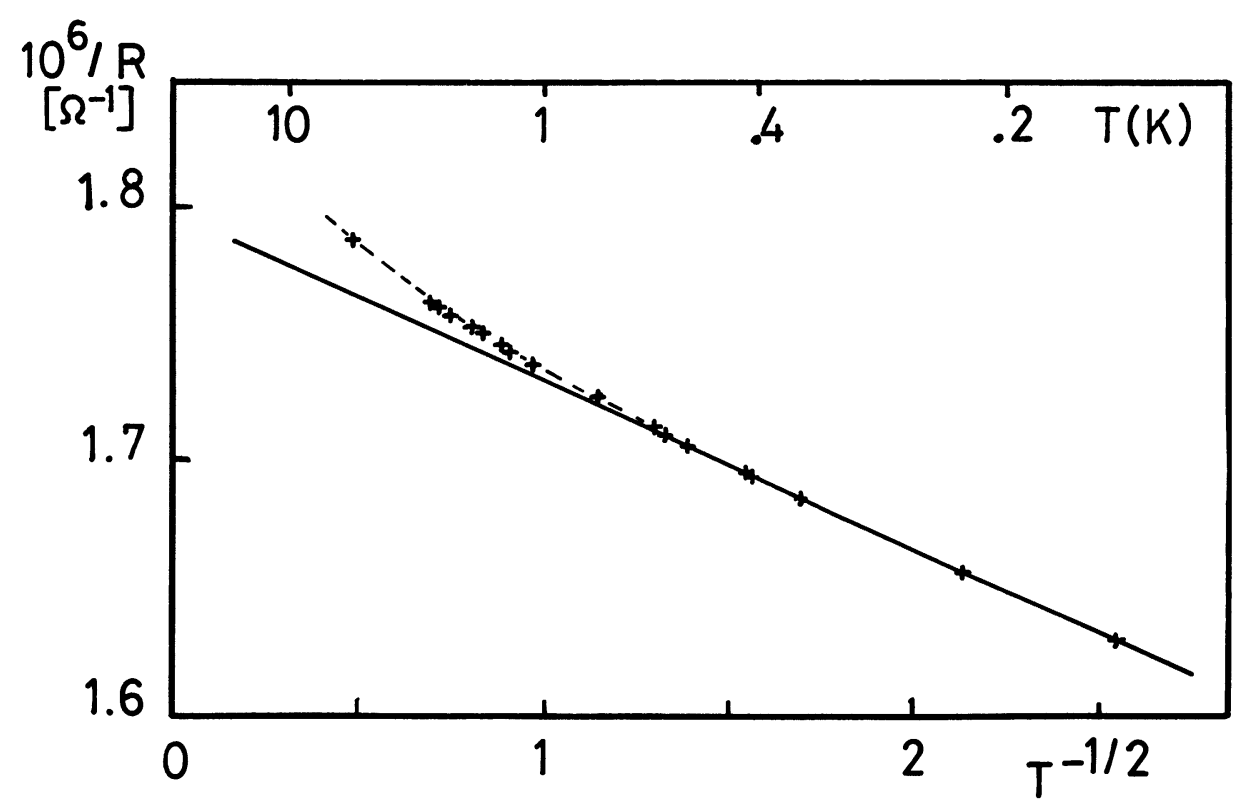

Fig. 4. - Conductance of the thinnest wire $(a)$ as a function of $T^{-1 / 2}$, characteristic of $1 \mathrm{~d}$ behaviour. 
The corresponding expression for the Anderson localization model in $1 \mathrm{~d}$ is :

$$
\delta C=-(\pi / 2 L)\left(e^{2} / \pi^{2} \hbar\right)\left(D \tau_{i}\right)^{1 / 2}
$$

which would imply $\tau_{i} \simeq 2 \times 10^{-10} / T(\mathrm{~s} / \mathrm{K})$; the order of magnitude agrees with the electronelectron inelastic times calculations [14] for thick films $\left(k_{\mathrm{F}} . t>1\right)$, and the predicted $(T \ln T)^{-1}$ temperature dependence is experimentally very close to the measured $T^{-1}$.

As observed in many different systems [5-9], correlation and localization effects coexist and have the same order of magnitude. The novel point in this work is to show the experimental observation of a continuous cross-over between a $1 \mathrm{~d}$ and a $2 \mathrm{~d}$ regimes. The effect, if clearly seen on the curves of figures 2 and 4, but a precise definition of a cross-over temperature is not a simple problem. For the time being, however, it is sufficient to remark that the cross-over is at about $0.5 \mathrm{~K}$ for a width of the wire $W \sim 1 \mu \mathrm{m}$. If one takes as cross-over condition

$$
W \sim\left(D \tau_{\text {inel }}\right)^{1 / 2}
$$

where the diffusion coefficient $D$ is related to the resistivity, one finds an inelastic relaxation time $\tau_{\text {inel }} \sim 10^{-9} \mathrm{~s}$ around $0.5 \mathrm{~K}$. This value is consistent with the fact that $\tau$ inelastic should be longer than the elastic relaxation time related to the bulk resistivity $\left(\sim 10^{-12} \mathrm{~s}\right)$, and with the values deduced from the analysis of the $1 \mathrm{~d}$ regime. For the broader samples $(b$ and $c)$, the cross-over is shifted to lower temperatures (Fig. 2), in agreement with the expected increase of the inelastic time as the temperature decreases.

The saturation effect below $100 \mathrm{mK}$ can be understood by writing that the current heats the electron gas and that the relaxation time between electrons and phonons is so long so that one has a relaxation bottleneck [15]. Comparison between $10 \mathrm{pA}$ points and higher currents values gives a variation of the electron phonon relaxation time $\tau$ roughly like $\dot{T}^{-p}$ with $p \sim 3$.

In conclusion, we have seen in semiconductor wires the same localization effects already found in very different systems and the cross-over from 1 to 2 dimensions; one can remark that here the Fermi wavelength is $400 \AA$, the elastic mean free path of the same order of magnitude and the Thouless localization length [16] $10 \mu \mathrm{m}$. The universality of the phenomenon is once more confirmed; the observation of cross-over between 1 and 2 dimensional regimes will help elucidate the condition for applicability of the models. The cross-over between dimensions already seen by Imry and Ovadyahu [17] in indium oxide samples in localization experiments, as well as in gallium arsenide in Shubnikov-de Haas measurements [8] is here shown between 1 and 2 dimensions as well as the dependence on the wire width.

\section{Acknowledgments.}

We thank Dr. D. Kaplan from L.C.R. Thomson-CSF for his help in designing the samples and for many discussions, Drs. P. Haen and J. L. Genicon from C.R.T.B.T. for higher temperature measurements, and Professor Y. Imry from Tel-Aviv University, Ramat-Aviv, for stimulating discussions.

This research has been made under contract 82.G.0500 of Ministère de la Recherche et de l'Industrie. 


\section{References}

[1] Abrahams, E., Anderson, P. W., Licciardello, D. C. and Ramakrishnan, T. V., Phys. Rev. Lett. 42 (1979) 673.

[2] Abrahams, E., Anderson, P. W. and Ramakrishnan, T. V., Philos. Mag. B 42 (1980) 827.

[3] Altshuler, B. L. and Aronov, A. G., Zh. Eksp. and Teor. Fiz. 77 (1979) 2028 ; Soviet Phys. JETP 50 (1979) 2028.

Altshuler, B. L., Khmelnitzkit, D., Larkin, A. I. and Lee, P. A., Phys. Rev. B 22 (1980) 5142.

[4] Fukuyama, H., J. Phys. Soc. Japan 50 (1981) 3407.

[5] Giordano, N., Phys. Rev. B 22 (1980) 5635.

[6] White, A. E., Tinkham, M., Skocpol, W. J. and Flanders, D. C., Phys. Rev. Lett. 48 (1982) 1752.

[7] Dynes, R. C., Garno, J. P. and Rowell, J. M., Phys. Rev. Lett. 40 (1982) 479.

Van den Dries, L., Van Haesendonck, Bruynseraede, Y., Deutscher, G., Phys. Rev. Lett. 46 (1981) 565.

Bergmann, G., Phys. Rev. B 25 (1982) 2937.

[8] Bishop, D. K., Tsui, D. C. and Dynes, R. C., Phys. Rev. Lett. 46 (1981) 360 ; Phys. Rev. B 26 (1982) 773.

WheEler, R. G., Phys. Rev. B 24 (1981) 4645.

Wheeler, R. G., Choi, K. K., Goel, A., Wisnieef, R., Prober D. E., Phys. Rev. Lett. 49 (1982) 1674.

[9] Dolan, G. J. and Osheroff, D. D., Phys. Rev. Lett. 43 (1979) 721.

[10] Fowler, A. B., Harstein, A. and WebB, R. A., Phys. Rev. Lett. 48 (1982) 196.

[11] Mort, N. F. and DAvis, E. A., Electronic Processes in Non-crystalline Materials (Clarendon Press, Oxford), 1971, p. 39.

[12] Sernelius, B. E. and Berggren, K. F., Philos. Mag. B 43 (1981) 115.

[13] Meyer, J. R. and Bartoli, F. J., Solid State Commun. 41 (1982) 19.

[14] Abrahams, E., Anderson, P. W., Lee, P. A., Ramakrishnan, T. V., Phys. Rev. B 24 (1981) 6783.

[15] Anderson, P. W., Abrahams, E. and Ramakrishnan, T. V., Phys. Rev. Lett. 43 (1979) 718.

[16] Thouless, D. J., Solid State Commun. 34 (1980) 683.

[17] Imry, Y. and Ovadyahu, Z., J. Phys. C 15 (1982) L327.

[18] Poole, D. A., Pepper, M., Berggren, K. F., Hill, G. and Myron, H. W., J. Phys. C 15 (1982) L21. 